3. K. Chandrasekharan and S. Minakshisundaram, Typical means, Tata Institute of Fundamental Research Monograph, Oxford Univ. Press, New York, 1952.

4. T. M. Flett, Some remarks on strong summability, Quart. J. Math. Oxford Ser. (2) 10 (1959), 115-139.

5. Pramila Srivastava, On strong Rieszian summability of infinite series, Proc. Nat. Inst. Sci. India, Part A 23 (1957), 58-71.

GorakhPUR UNIVERSITy, GorakhPUR, INDIA

\title{
ON HYPONORMAL OPERATORS
}

\section{H. SHETH}

1. An operator $T$ defined on a Hilbert space $H$ is said to be hyponormal if $T^{*} T-T T^{*} \geqq 0$, or equivalently if $\left\|T^{*} x\right\| \leqq\|T x\|$ for every $x \in H$. An operator $T$ is said to be seminormal if either $T$ or $T^{*}$ is hyponormal. If $T$ is hyponormal, then $T-z I$ is also hyponormal for all complex values of $z$.

The spectrum of an operator $T$, in symbols $\sigma(T)$, is the set of all those complex numbers $z$ for which $T-z I$ is not invertible. A complex number $z$ is said to be an approximate proper value for the operator $T$ in case there exists a sequence $x_{n}$ such that $\left\|x_{n}\right\|=1$ and $\left\|(T-z I) x_{n}\right\|$ $\rightarrow 0$. The approximate point spectrum of an operator $T$, in symbols $\Pi(T)$, is the set of approximate proper values of $T$. The numerical range of an operator $T$, denoted by $W(T)$, is the set defined by the relation

$$
W(T)=\{(T x, x):\|x\|=1\} .
$$

$\mathrm{Cl}(W(T))$ will, as usual, denote the closure of $W(T)$. An operator $S$ is said to be similar to an operator $T$ in case there exists an invertible operator $A$ such that $S=A^{-1} T A$.

In this note, all the operators will relate to a Hilbert space $H$.

We shall prove the following theorem.

THEOREM. Let $N$ be a hyponormal operator. If for an arbitrary operator $A$, for which $0 \notin \mathrm{Cl}(W(A)), A N=N^{*} A$, then $N$ is self-adjoint.

Received by the editors April 11, 1966. 
For proving this theorem, we need certain results which we formulate in the form of lemmas.

2. Lemma 1. Let $T$ be a hyponormal operator and let $z_{1}, z_{2} \in \Pi(T)$, $z_{1} \neq z_{2}$. If $x_{n}$ and $y_{n}$ are the sequences of unit vectors of $H$ such that $\left\|\left(T-z_{1} I\right) x_{n}\right\| \rightarrow 0$ and $\left\|\left(T-z_{2} I\right) y_{n}\right\| \rightarrow 0$, then $\left(x_{n}, y_{n}\right) \rightarrow 0$.

Proof. See [1, p. 170].

Lemma 2. If $T$ is hyponormal, $\sigma\left(T^{*}\right)=\Pi\left(T^{*}\right)$.

Proof. See [2].

Lemma 3. If $T$ is a hyponormal operator such that $\sigma(T)$ is a set of real numbers, then $T$ is self-adjoint.

Proof. See [3, Theorem 4, Corollary 1].

Lemma 4. If an operator $A$ is similar to an operator $B$, then $A$ is bounded below iff $B$ is bounded below. In other words if $A$ and $B$ are similar, then $\Pi(A)=\Pi(B)$.

Proof. Let $A=T^{-1} B T$ for an invertible operator $T$. Now if $B$ is bounded below, then $B^{*} B \geqq \alpha I$ for some constant $\alpha>0$. Since $T$ is invertible, there exist constants $\beta>0$ and $\gamma>0$ such that $T^{*} T \geqq \beta I$ and $\left(T T^{*}\right)^{-1}=T^{*-1} T^{-1} \geqq \gamma I$.

Now $\quad A^{*} A=T^{*} B^{*} T^{*-1} T^{-1} B T=(B T)^{*} T^{*-1} T^{-1} B T \geqq(B T)^{*} \gamma I B T$ $=\gamma T^{*} B^{*} B T \geqq \gamma T^{*} \alpha I T=\alpha \gamma T^{*} T \geqq \alpha \beta \gamma I$ i.e. $A$ is bounded below. Since the above process is reversible, the stated result follows.

The relation $\Pi(A)=\Pi(B)$ follows from the following two observations.

(i) If $A$ is similar to $B$, then $A-z I$ is similar to $B-z I$ for all complex numbers $z$.

(ii) $z \notin \Pi(A)$ iff $A-z I$ is bounded below.

3. Proof of the theorem. Since $0 \notin \mathrm{Cl}(W(A)), A$ is invertible. Hence $N=A^{-1} N^{*} A$ and it follows from Lemmas 2 and 4 that $\sigma(N)$ $=\sigma\left(N^{*}\right)=\Pi\left(N^{*}\right)=\Pi(N)$.

In order to complete the proof of the theorem, it is sufficient, by virtue of Lemma 3, to prove that $\sigma(N)$ is real. Suppose on the contrary that there exists a $z \in \sigma(N)$ such that $z \neq \bar{z}$. Since $z \in \sigma(N)$ $=\Pi(N)$, there exists a sequence $x_{n}$ of unit vectors such that $\left\|\left(N^{*}-\bar{z} I\right) x_{n}\right\| \leqq\left\|(N-z I) x_{n}\right\| \rightarrow 0$.

Since $0 \notin \mathrm{Cl}(W(A))$, the relation $\left\|\left(N^{*}-\bar{z} I\right) x_{n}\right\|=\left\|\left(A N A^{-1}-\bar{z} I\right) x_{n}\right\|$ $=\left\|A(N-\bar{z} I) A^{-1} x_{n}\right\| \rightarrow 0$ implies that $\left\|(N-\bar{z} I) A^{-1} x_{n}\right\| \rightarrow 0$. Hence $\left(x_{n}, \quad A^{-1} x_{n}\right)=\left(A A^{-1} x_{n}, \quad A^{-1} x_{n}\right) \rightarrow 0$ by Lemma 1. Put $y_{n}$ 
$=A^{-1} x_{n} /\left\|A^{-1} x_{n}\right\|$, then $\left\|y_{n}\right\|=1$ and $\left(A y_{n}, y_{n}\right) \rightarrow 0$ i.e. $0 \in \mathrm{Cl}(W(A))$, a contradiction. This completes the proof of the theorem.

We deduce, as a corollary, the following result.

COROllari. Let $N$ be a seminormal operator. If for an arbitrary operator $A$, for which $0 \notin \mathrm{Cl}(W(A)), A N=N^{*} A$, then $N$ is self-adjoint.

Proof. Suppose that $N^{*}$ is hyponormal. The proof of the theorem shows that $0 \notin \mathrm{Cl}(W(A))$ implies $0 \notin \mathrm{Cl}\left(W\left(A^{-1}\right)\right)$. Now $A N=N^{*} A$ implies $A^{-1} N^{*}=N A^{-1}$ i.e. $B M=M^{*} B$, where $M=N^{*}$ is hyponormal and $0 \notin \mathrm{Cl}(W(B))=\mathrm{Cl}\left(W\left(A^{-1}\right)\right)$. Hence $M=M^{*}$ by the theorem i.e. $N=N^{*}$.

The author expresses his thanks to Professor U. N. Singh and Professor S. K. Berberian for their comments and suggestions and to the C.S.I.R. of India for the award of a Junior Fellowship.

\section{REFERENCES}

1. S. K. Berberian, Introduction to Hilbert space, Oxford Univ. Press, New York, 1961.

2. - A note on hyponormal operators, Pacific J. Math. 12 (1962), 1871-1875.

3. J. G. Stampfli, Hyponormal operators and spectral density, Trans. Amer. Math. Soc. 117 (1965), 469-476.

M. S. University of BAROdA, INDiA 